\title{
Effect of Molecular Rotational Degrees of Freedom on Mechanical and Thermodynamic Properties of Solid Methane at Temperatures above $50 \mathrm{~K}$
}

\author{
Antonina V. Leont'eva, ${ }^{1}$ Andrew Yu. Prokhorov, ${ }^{1}$ \\ Anatoly Yu. Zakharov, ${ }^{2}$ and Alexander I. Erenburg ${ }^{3}$ \\ ${ }^{1}$ Donetsk Institute for Physics and Engineering, NAS of Ukraine, Luxemburg Street 72, Donetsk 83114, Ukraine \\ ${ }^{2}$ Novgorod State University, Great Sankt Peterburg Street 41, Velikiy Novgorod 173003, Russia \\ ${ }^{3}$ Ben-Gurion University of Negev, P.O. Box 653, 8410501 Beer-Sheva, Israel \\ Correspondence should be addressed to Alexander I. Erenburg; erenburga@gmail.com
}

Received 19 June 2014; Revised 22 August 2014; Accepted 8 September 2014; Published 7 October 2014

Academic Editor: Tatyana Sizyuk

Copyright (c) 2014 Antonina V. Leont'eva et al. This is an open access article distributed under the Creative Commons Attribution License, which permits unrestricted use, distribution, and reproduction in any medium, provided the original work is properly cited.

The paper presents an analysis of extensive data set of mechanical, structural, thermophysical, and spectral properties of solid methane in the temperature interval $0.5 \cdot T_{\mathrm{tr}}-T_{\mathrm{tr}}\left(T_{\mathrm{tr}}\right.$ is the triple point temperature) under equilibrium vapor pressure. It is shown that the anomalies of the studied properties (or lack of reliable data) at temperatures $60-70 \mathrm{~K}$ have been observed in the body of the reviewed papers. We proposed that the observed anomalies are due to a transition between classical and quantum regimes of collective rotational degrees of freedom of methane molecules in this temperature interval.

\section{Introduction}

The development of far cosmos research and discovery of atmospheres at some planets of the Solar System have inspired new large interest to mechanical and thermodynamic properties of solidified gases, in particular, solid methane [1].

Solid methane is the lightest representative among the group of the simplest molecular crystals, formed by $\mathrm{CX}_{4}$ tetrahedral molecules with $4-3 \mathrm{~m}$ symmetry [2], where $\mathrm{X}$ is hydrogen isotopes or halogen atoms $\mathrm{F}, \mathrm{Cl}$, and so forth or their isotopes.

In this paper, we present a detailed analysis of the experimental data of many authors on mechanical, structural, thermophysical, and spectral properties of solid methane in temperature interval $0.5 \cdot T_{\mathrm{tr}}-T_{\mathrm{tr}}\left(T_{\mathrm{tr}}\right.$ is the triple point temperature) under equilibrium vapor pressure. The body of reviewed papers shows the presence of anomalies of above properties at temperatures $60-70 \mathrm{~K}$, or the reliable data are absent. We suppose that these anomalies are caused by a sudden change of rotational degrees of freedom of $\mathrm{CH}_{4}$ molecules at temperatures between 60 and $70 \mathrm{~K}$ [3].

\section{Some Data on Thermodynamic Properties of Solid Methane}

Methane is the most examined crystal of $\mathrm{CX}_{4}$ group. The well-known reviews $[4,5]$ have presented extensive data on thermodynamic, structural, and mechanical characteristics of solid methane. However, the anomalies of a series of solid methane properties at temperatures higher than $0.5 T_{\text {tr }}$ were not mentioned in these reviews.

Under equilibrium vapor pressure, crystalline methane (triple point temperature $T_{\mathrm{tr}}=90.67 \mathrm{~K}$ [4]) undergoes a $\lambda$ type phase transition at $T_{\lambda}=20.48 \mathrm{~K}$ [3]. In both phases, low-temperature II and high-temperature I, carbon atoms form a FCC lattice (space group Fm3m [4]). It is commonly supposed that in low-temperature phase carbon atoms are ordered in the lattice (expected space group Fm3c or P4-3m 
[4]) and make certain librational vibrations relative to the center of inertia of methane molecule and also reorientational hopping from one equilibrium position to another. In phase I, hydrogen atoms entirely loose the ordering and make "hindered spherical rotation" relative to the gravity center of hydrogen atom. Volume jump at the phase transition I-II is about $0.3 \%$ [4].

\section{Essential Experimental Data: Analysis and Discussion}

The problem of the change of methane molecule's rotational state at temperatures $60-70 \mathrm{~K}$ was firstly established by Tomita [3]. In this paper, he studied NMR spectra of solid methane and had revealed that (i) half width of resonance absorption lines $H_{1 / 2}$ decreases suddenly (see Figure 1) and (ii) time of spin-lattice relaxation changes essentially at temperatures below $60 \mathrm{~K}$ (see Figure 2). Accounting of the above-mentioned facts that in a narrow range of temperatures the activation energy of rotation of the methane molecule is increased by about twenty times, Tomita came to the conclusion that after $60 \mathrm{~K}$ there is a transition from the hindered rotation of methane to almost free. We can suggest that at temperatures above $60 \mathrm{~K}$ the methane molecules turn to a new rotational state, namely, to essentially free (or slightly hindered) rotation such as molecular behavior in liquid phase [6]. In this case, the noncentral intermolecular interaction is almost absent and the contribution of van der Waals central forces becomes dominant in the intermolecular interaction.

Note that this transformation of rotational behavior of methane molecules is displayed not only in the NMR parameters.

Figure 3(a) shows the temperature dependence of low frequency internal friction (LFIF) of solid methane $Q^{-1}$ that has been studied by an inverse torsion pendulum method at frequencies $5-10 \mathrm{~Hz}$ and magnitude of deformation 5 . $10^{-5}$. Figure 3(b) presents the temperature dependence of the dynamic shear modulus $G$ derived from the frequency of torsion oscillations $f\left(f^{2} \sim G\right)$ [7]. Procedure of preparing of optically transparent solid $\mathrm{CH}_{4}$ specimens of high quality with grain size $0.5-2 \mathrm{~mm}$ with free surface and the experimental setup for cryocrystals studying by LFIF method have been reported in [7-9].

It is seen from Figure 3(a) that the key feature of the LFIF spectrum $Q^{-1}(T)$ for a whole temperature range is a high peak near $65 \mathrm{~K}$. Its magnitude reaches the value $10^{-1}$ that is by a factor of about ten times higher than the magnitude of the peak near temperature of the phase I-II transition. Such anomalous rise of $Q^{-1}$ in $\mathrm{CH}_{4}$ cannot be explained in the frameworks of conventional classic conception. It is necessary to note that such great LFIF peaks have not observed in the previous studies of cryocrystals with central intermolecular forces, for example, in solid argon [8].

Note that the sharp $Q^{-1}$ anomaly is similar to the behavior of LFIF spectra in solid oxygen [9]. This allows us to suggest that at $T \approx 65 \mathrm{~K}$ the phase transition of latent form in solid methane occurs. It is associated with an abrupt change of the rotational state of $\mathrm{CH}_{4}$ molecules [10]. Later it has been

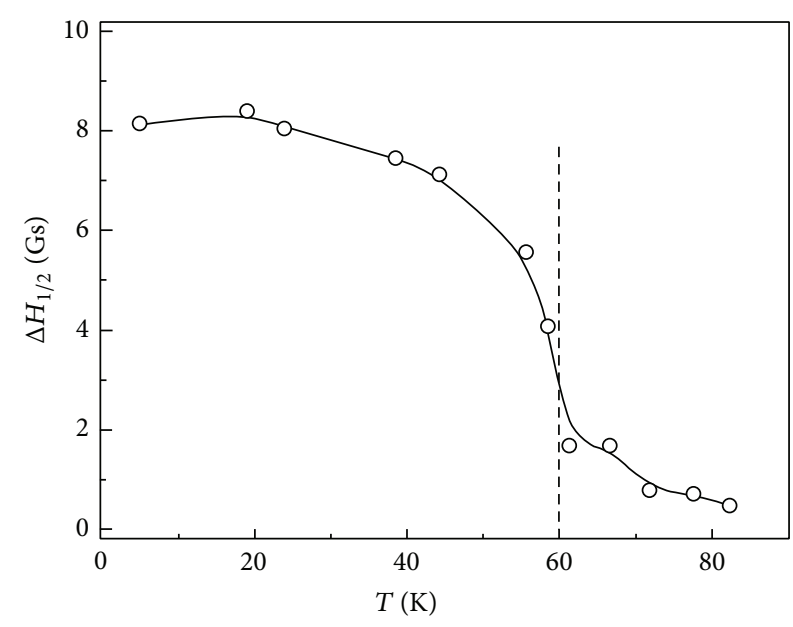

FIgURE 1: Temperature dependence of half width of resonance absorption lines $H_{1 / 2}$ of the dynamical local field [3].

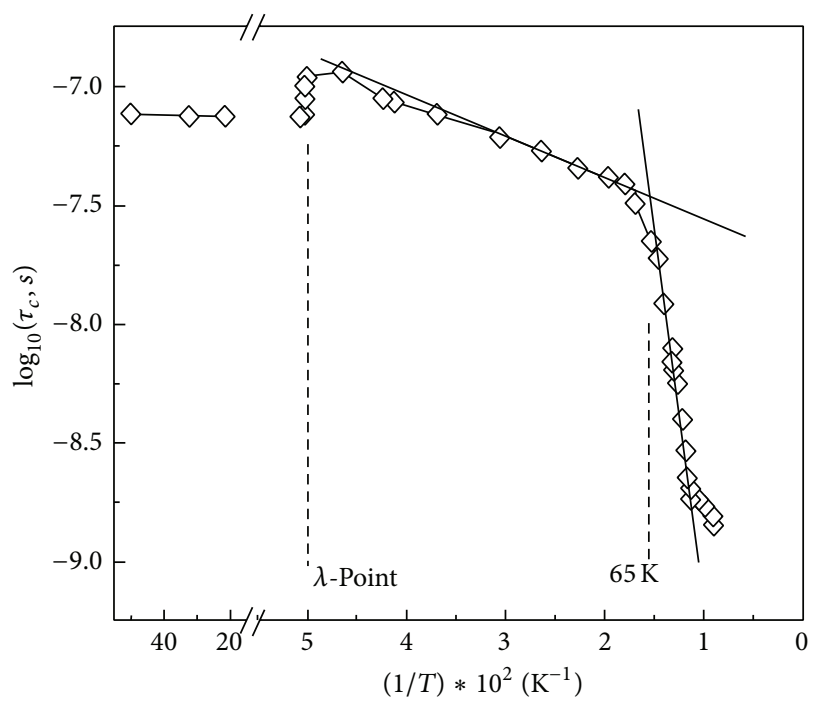

Figure 2: The dependence of characteristic time $\tau_{c}$ of spin-lattice relaxation on reciprocal temperature [3].

shown that a set of the observed anomalies can be explained by existence of collective rotational degrees of freedom in solid methane [11].

It is necessary to note that [11] does not provide a report on rotation of separate methane molecules. With the lowering of temperature a collectivization of the rotational degrees of freedom of methane molecules occurs with a new effective moment of inertia of corresponding quasi-particles (topon) at gradual transition of a crystal to the quantum tunnel state of the rotational degrees of freedom of methane molecules occurs with a new effective moment of inertia of corresponding quasi-particles (topons) at gradual transition of a crystal to the quantum tunnel state.

The temperature dependence of $f^{2}(T)$ also shows a clearly defined minimum near $65 \mathrm{~K}$ indicating a significant alteration of intermolecular forces in a narrow temperature 


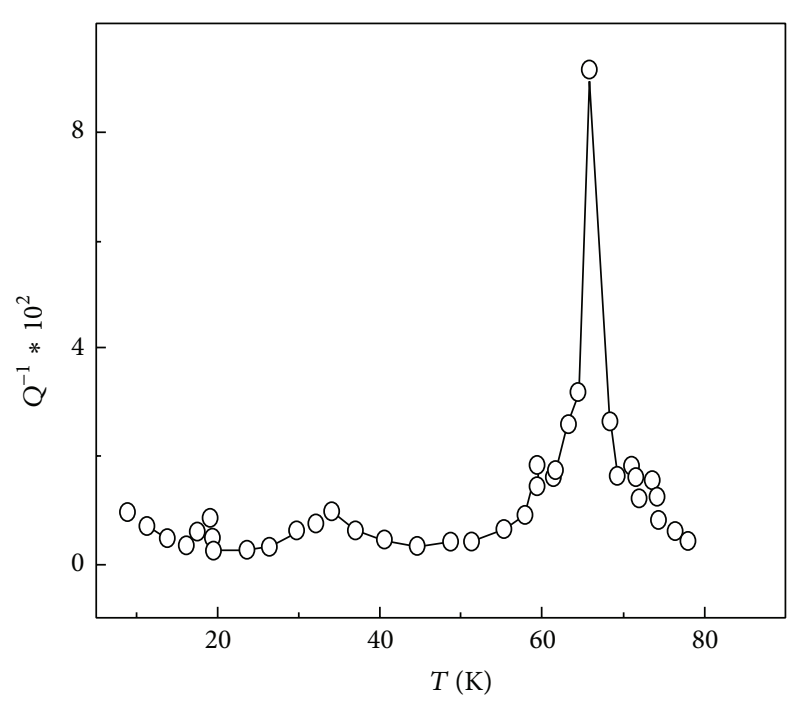

(a)

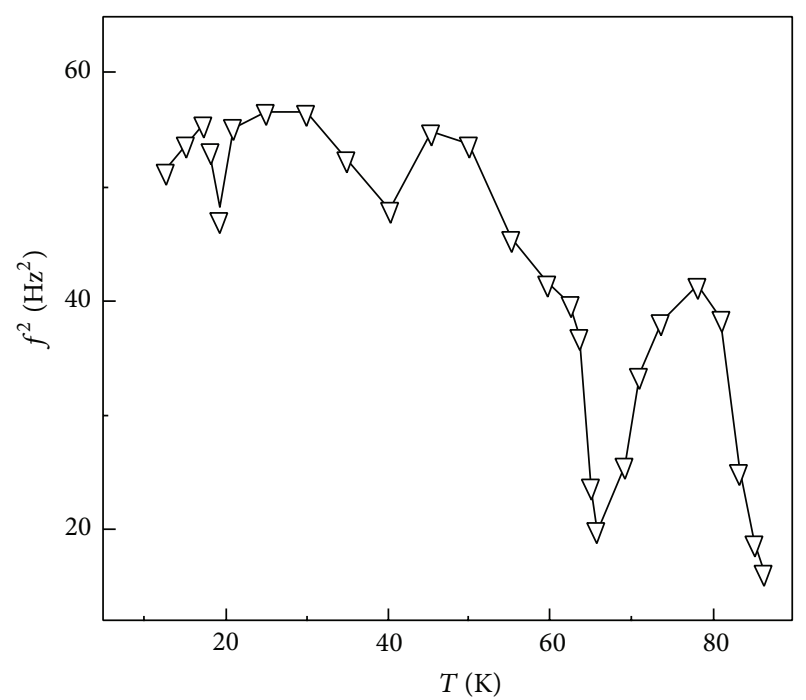

(b)

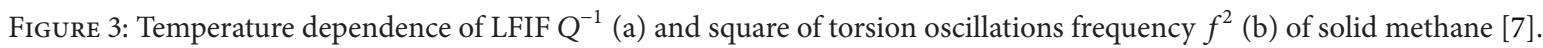

interval $(3-5 \mathrm{~K})$ (Figure $3(\mathrm{~b})$ ). This also can be related with the abovementioned change of rotational movement of $\mathrm{CH}_{4}$ molecules in temperature interval $60-70 \mathrm{~K}$ that affects the mechanical characteristics of solid methane.

Such a conclusion is in agreement with the results of recent work [1] which is devoted to study of adhesion and plasticity of polycrystalline methane in temperature interval $10-90 \mathrm{~K}$. The authors have shown that the adhesion is strong in temperature range $50-90 \mathrm{~K}$ where solid methane is soft and sticky. Towards melting temperature, solid methane behaves as very viscous non-Newtonian liquid, but at temperatures below $30 \mathrm{~K}$ it loses its "stickiness and plasticity" and behaves like an ordinary glass.

However, despite the mentioned temperature interval 50$90 \mathrm{~K}$ of softness and plasticity of solid methane, in the same work [1], after studying of temperature dependence of shear corresponding to a connection breaking point between the solid methane and the special probes, it has been revealed that the shear stress is raised with temperature up to $60 \mathrm{~K}$ and then rapidly decreases with temperature approaching to the melting point (see Figure 6 in [1]). This fact directly indicates the alteration of intermolecular forces character in solid methane that is possibly associated with the transition of methane molecules to a new rotational state at temperatures above $60 \mathrm{~K}$.

Analysis of the available experimental data on various properties of solid methane shows that the change of rotational movement of methane molecules is displayed not only in the abovementioned NMR and mechanical characteristics.

Thus, for example, anomalies of elastic properties of solid methane in temperature interval $60-70 \mathrm{~K}$ have been reported in $[12,13]$. Figure 4 shows that anomalies of the temperature dependence of longitudinal sound velocity $v_{l}(T)$ have been observed not only near the phase transition but also at temperatures near $60 \mathrm{~K}$. Similar peculiarities have been observed in [13] for the velocities of both longitudinal $v_{l}$ and transverse $v_{s}$ sound (see Figure 5).

It is important to note that although the paper [12] is mentioned in the reviews $[4,5]$, but nothing more, its results have not been analyzed. As concerns the paper [13], its results have been cited in the reviews both for velocity of longitudinal and transverse sound. The peculiarities of sound velocity [13] are clearly seen in corresponding figures of $[4,5]$ like as in Figure 5 of this work. Nevertheless, neither authors of [13] nor those of $[4,5]$ have not considered the anomalous behavior of the temperature dependence of sound velocity near $T=65 \mathrm{~K}$.

It should be emphasized that, as distinct from mechanical properties, the anomalies of thermodynamical properties of solid methane in analyzed temperature range are expressed more weakly.

Figure 6 presents the temperature dependencies of molar volume $V_{m}$ of solid methane reported in [14-16] by several independent research groups. It is clearly seen that $V_{m}(T)$ [14] shows a peculiarity near $60 \mathrm{~K}$, and jointing of the data $[15,16]$ yields practically a repetition of $V_{m}(T)$ from [14]. Anomalies of $V_{m}(T)$ slightly exceed an experimental error of [14-16]. Even though they are small, they also can be associated with the transition of $\mathrm{CH}_{4}$ molecules to a new rotational state.

Figure 7 shows the temperature dependencies of the coefficient of volume expansion $\beta(T)$ according to $[15,17-$ 19]. It is seen that the data divergence of various papers exceeds the possible experimental errors. Demating of the results $[15,19]$ is similar to the peculiarities observed in $V_{m}(T)$ behavior. Quite possible is that this demating is also related to the above alteration of rotational state of methane molecules. It is interesting to note that since X-ray results [17] do not show any visible feature of $\beta(T)$ however the authors of [17] have observed a certain rise of $\beta(T)$ at temperatures above $70 \mathrm{~K}$ in comparison with the estimated dependence for ideal lattice (see Figure 3 in [17]). Besides, it is seen in 


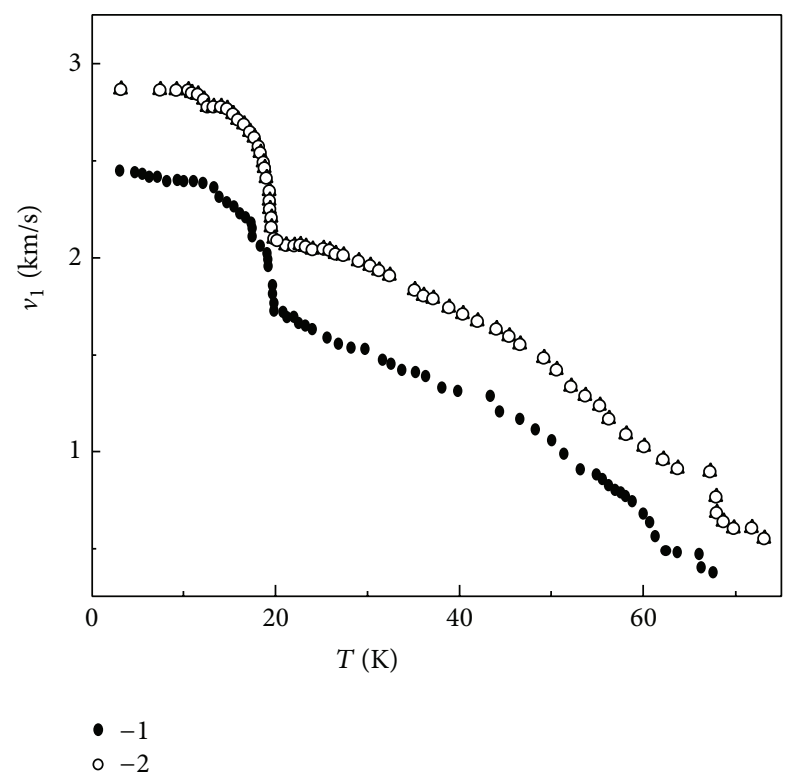

Figure 4: Temperature dependence of longitudinal sound velocity $v_{l}[12]$ for the samples prepared at various growth rates: 1 : fast growth and 2: slow growth.

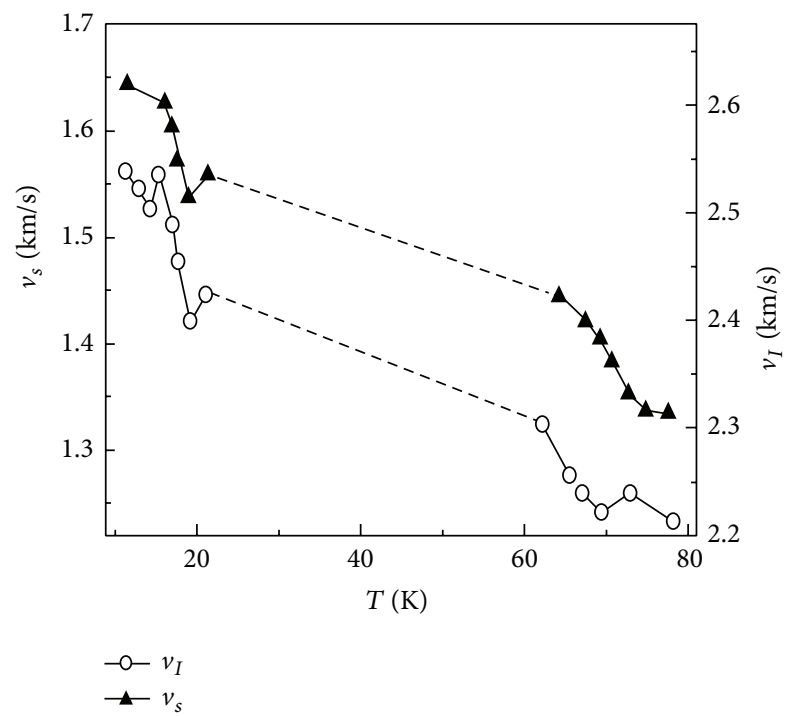

FIGURE 5: Temperature dependence of the velocity of longitudinal $v_{l}$ and transverse $v_{s}$ sound of solid methane [13] in the vicinity of the phase transition temperature and in temperature interval $60-80 \mathrm{~K}$.

Figure 7 that, at temperatures above $70 \mathrm{~K}, \beta(T)$ obtained from dilatometric measurements [17] rises faster compared to $\beta(T)$ obtained from X-ray data of [19]. Difference between the data reaches $15 \%$ at temperature $89 \mathrm{~K}$ (see Table 8.10 in [4]). The authors explain it by point defects arising in the sample just as it was observed in solidified inert gases (i.e., Ar and $\mathrm{Kr}$ ) at premelting temperatures since "solid methane at high temperatures is the closest to crystalline inert gases" [17]. The authors have emphasized that at this temperature range $(T>70 \mathrm{~K})$ "a symmetrical distinction between inert

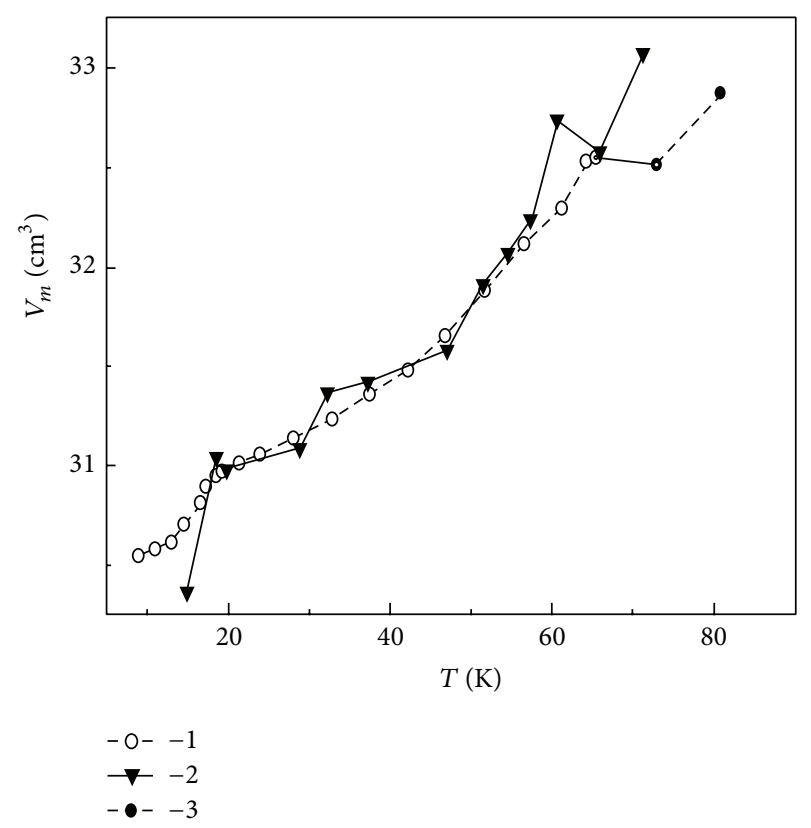

FIgURE 6: Temperature dependencies of molar volume for solid methane according to [14-16]: 1: [15], 2: [14], and 3: [16].

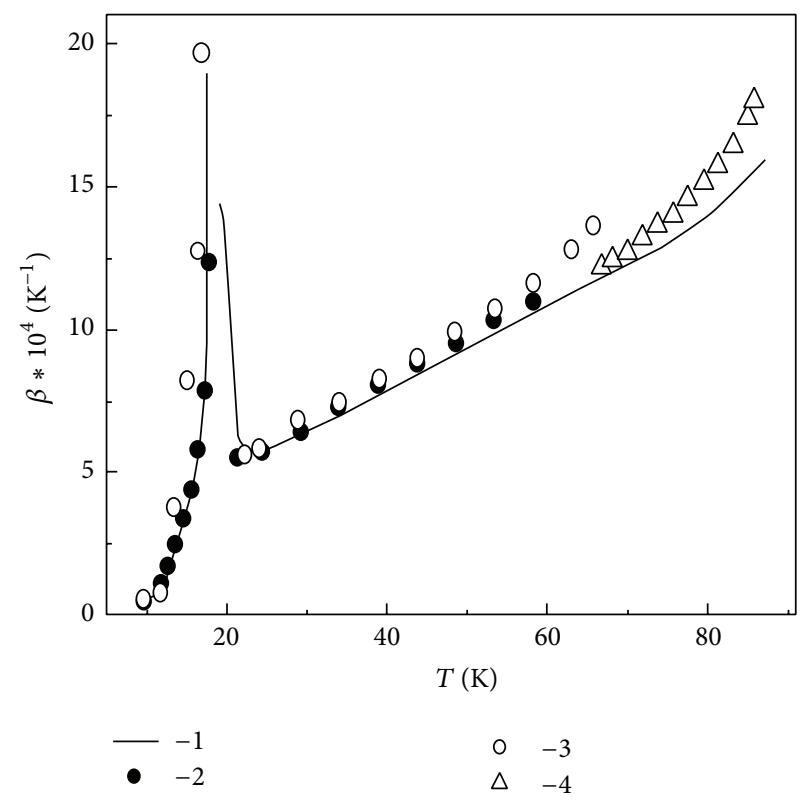

Figure 7: Temperature dependencies of volume coefficient of thermal expansion $\beta$ for solid methane according to the data of several papers: 1-[17]; 2-[18]; 3-[15]; 4-[19].

gases atoms and the methane molecules is absent since the last make a practically free rotation in the lattice" [17].

Some more complex situation arises when analyzing the temperature dependence of heat capacity $C_{p}(T)$. In the analyzed temperature interval $\left(0.5 \cdot T_{\mathrm{tr}}-T_{\mathrm{tr}}\right)$, only data of three papers are available [20-22]. Two earlier of them $[20,21]$ had not reported any noticeable peculiarities in $C_{p}(T)$ behavior. But the latest paper [22] which reports the temperature 
dependencies of heat capacity for both $\mathrm{CH}_{4}$ and $\mathrm{CD}_{4}$ has revealed a certain mismatch between these dependencies (see Figure 8).

It has to be noted that, in contrary to $\mathrm{CH}_{4}, \mathrm{CD}_{4}$ can exist in three phases under equilibrium vapor pressure [4]. High temperature phase I and middle one II are similar to the phases I and II of solid methane. Low temperature phase III of $\mathrm{CD}_{4}$ is characterized by more complex ordering of $\mathrm{D}$ atoms relatively to centre of mass of carbon atoms accompanied by lowering of symmetry (space group P- $4 \mathrm{~m} 2$ or P $42 / \mathrm{mbc}[4]$ ). Therefore, $\mathrm{CD}_{4}$ molecules in phase I, such as methane ones, make a hindered rotation around a centre of mass of carbon atoms. It is seen from Figure 8 that $C_{p}(T)$ data cover a whole temperature range of phase I and do not show any features.

In temperature interval $30-90 \mathrm{~K}$ the behavior of $C_{p}$ is less clear. Inset in Figure 8 shows the $C_{p}(T)$ dependencies for both methane and deuteromethane. It can be seen that the data for $\mathrm{CD}_{4}$ are continuous over all temperature interval, but the $\mathrm{CH}_{4}$ data are absent between 61 and $85 \mathrm{~K}$. Besides, this lack is not commented in [22].

Therefore, the data on $\mathrm{CH}_{4}$ heat capacity are not in contradiction with the above assumption about cooperative behavior of rotational degrees of freedom of methane molecules. The observed anomalies are caused by a transition of the cooperative excitations quasiparticles from quantum (tunnel) to classical (thermoactivation) behavior.

In regard to $\mathrm{CD}_{4}$, it should be noted that moment of inertia of a $\mathrm{CD}_{4}$ molecule is twice as much as for methane molecule. This leads to the change of temperature interval of the transition. Because of lack of a microscopical theory allowing establishing a relation between moment of inertia of a single molecule and effective moments of inertia of collective excitations (topons), this temperature interval cannot be determined theoretically.

\section{Conclusions}

The presented analysis of available experimental data about the mechanical, structural, thermophysical, and elastic properties of solid methane in temperature range above $0.5 \cdot T_{\text {tr }}$ under equilibrium vapor pressure shows that most of considered papers report the anomalies of the studied properties at temperatures $60-70 \mathrm{~K}$ or have not the reliable data at this temperature range.

As it follows from [11], a transition of the collective excitations of methane molecular degrees of freedom from quantum behavior to classic one occurs in this temperature interval. Tomita [3] has interpreted such a process as the change of activation energy of rotational state of methane molecules at temperatures $60-70 \mathrm{~K}$.

Just this transition of collective rotational degrees of freedom of methane molecules from quantum behavior to classical one affects the NMR and LFIF spectra as well as the mechanical properties of solid methane. More weakly this effect is displayed in temperature dependencies of thermodynamic parameters of solid methane.

Thus, the change of rotational state of the methane molecules in temperature interval $60-70 \mathrm{~K}$ must considerably

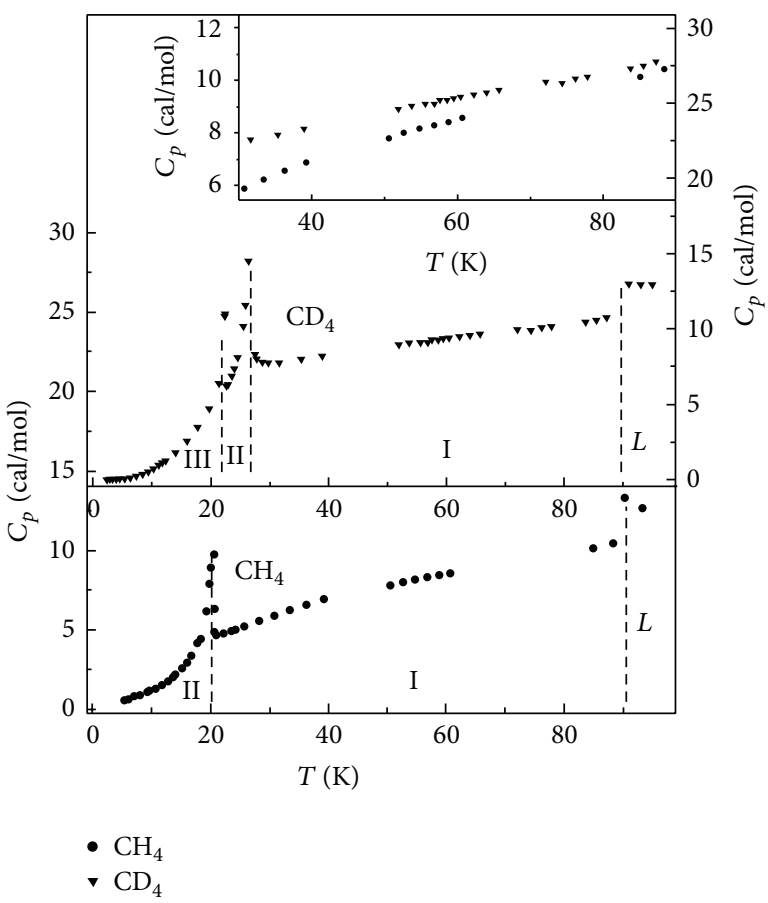

Figure 8: Temperature dependencies of heat capacity $C_{p}(T)$ for $\mathrm{CH}_{4}$ and $\mathrm{CD}_{4}$ according to [22].

weaken the intermolecular forces in solid methane. This, in turn, affects its mechanical characteristics. Therefore, results of this paper may be of great importance when analyzing the astrophysical data about the surface of Solar system planets and their satellites.

\section{Conflict of Interests}

The authors declare that there is no conflict of interests regarding the publication of this paper.

\section{Acknowledgments}

The authors express their great gratitude to the Academician of National Academy of Sciences of Ukraine, Vadim G. Manzhelii, and Professors L. P. Mezhov-Deglin, E. P. Rudavskii, and Yu. A. Mamalui for the useful discussion and important advices. Anatoly Yu. Zakharov is grateful to the Russian Ministry of Science and Education for partial financial support within the framework of state demand.

\section{References}

[1] O. Kirichek, A. J. Church, M. G. Thomas et al., "Adhesion, plasticity and other peculiar properties of solid methane," Cryogenics, vol. 52, no. 7-9, pp. 325-330, 2012.

[2] H. Gerhard, Molecular Spectra and Molecular Structure. Part II. Infrared and Raman Spectra of Polyatomic Molecules, Krieger, 1989. 
[3] K. Tomita, "States of solid methane as inferred from nuclear magnetic resonance," Physical Review, vol. 89, no. 2, pp. 429438, 1953.

[4] V. G. Manzhelii, A. I. Prokhvatilov, V. G. Gavrilko, and A. P. Isakina, Structure and Thermodynamic Properties of Cryocrystals: Handbook, Begell House, New York, NY, USA, 1998.

[5] A. I. Prokhvatilov, Plasticity and Elasticity of Cryocrystals (Handbook), Begel Haus, New York, NY, USA, 2001.

[6] R. G. Gordon, "Molecular motion in infrared and raman spectra," The Journal of Chemical Physics, vol. 43, no. 4, pp. 13071310, 1965.

[7] A. V. Leonteva, G. A. Marinin, A. Y. Prokhorov, and B. Ya. Sukharevskii, "Anomalies of low-frequency internal friction in crystalline methane," Fizika Nizkikh Temperatur, vol. 20, pp. 815-819, 1994.

[8] A. V. Leont'eva, G. A. Marinin, and I. A. Oberemchenko, "Lowtemperature internal friction in crystalline argon," Journal Fizika Nizkih Temperatur (Soviet Journal of Low Temperature Physics), vol. 10, pp. 1279-1284, 1984.

[9] A. I. Erenburg, A. V. Leont'eva, V. N. Varyukhin, G. A. Marinin, and A. Yu. Prokhorov, "The internal friction and phase transition of solid oxygen," Fizika Nizkikh Temperatur, vol. 37, no. 5, pp. 539-542, 2011.

[10] A. V. Leont'eva, A. Y. Prokhorov, and A. I. Erenburg, "On the issue of second phase transition in solid methane," in Proceedings of the 9th International Conference on Cryocrystals and Quantum Crystals (CC '12), Thesis, p. 46, Odessa, Ukraine, 2012.

[11] A. Y. Zakharov, A. V. Leontieva, A. Y. Prokhorov, and A. I. Erenburg, "Anomalous properties of crystalline methane in temperature interval between 60 and 70K," Physics of the Solid State, vol. 56, pp. 1501-1505, 2014.

[12] F. A. Stahl, R. P. Wolf, and M. B. Simmonds, "Ultrasound propagation in solid methane," Physics Letters A, vol. 27, no. 8, pp. 482-483, 1968.

[13] P. A. Bezuglii, N. G. Burma, and R. H. Minyafayev, "The elastic constant of the polycrystalline methane in the temperature range 14.4-77 K," Fizika Tverdogo Tela (Soviet physics, Solid state), vol. 8, pp. 744-749, 1966.

[14] S. C. Greer and L. Meyer, "The crystal structure and thermal expansion of solid methane," Zeitschrift für Angewandte Physik, vol. 27, pp. 198-199, 1969.

[15] A. I. Prokhvatilov, V. M. Gasan, and A. I. Erenburg, "Structure and thermal expansion of solid methane," in Fizika Kondensirovannogo Sostoyaniya, no. 10, pp. 135-153, Institute for Low Temperature Physics and Engineering, USSR, Kharkiv, Ukraine, 1970.

[16] A. M. Tolkachev and V. G. Manzhelii, "Density of ammonia and methane in solid state," Fizika Tverdogo Tela (Soviet physics, Solid State), vol. 5, pp. 2506-2010, 1964.

[17] A. I. Prokhvatilov and A. P. Isakina, "Lattice parameters, thermal expansion and density of vacancies in solid $\mathrm{CH}_{4}$," Fizika Nizkih Temperatur, vol. 9, pp. 419-428, 1983.

[18] V. G. Manzhelii, A. M. Tolkachev, and V. G. Gavrilko, "Thermal expansion of solid $\mathrm{CH}_{4}$ and $\mathrm{CD}_{4}$," Journal of Physics and Chemistry of Solids, vol. 30, no. 12, pp. 2759-2763, 1969.

[19] V. G. Manzhelii, L. M. Tarasenko, A. I. Bondarenko, and V. G. Gavrilko, "The coefficients of thermal expansion, and the sound velocity in the crystalline methane near the melting temperature," Fizika Tverdogo Tela (Soviet physics, Solid state), vol. 17, pp. 2259-2264, 1975.
[20] K. P. F. Clusius, "Uber die speeifische Warme einiger kondensierter Gase zwischen 10 Grad abs. und ihrem triple Punkt," Zeitschrift für Physikalische Chemie (Leipzig), vol. 83, pp. 41-79, 1929.

[21] K. P. A. Clusius and A. Perlick, "Die Unstetigkeit im thermischen und kalorischen Verhalten des Methans bei $20,4^{\circ}$ abs. als Phasenumwandlung 2. Ordnung," Zeitschrift für Physikalische Chemie B, vol. 24, pp. 313-327, 1934.

[22] J. H. Colwell, E. K. Gill, and J. A. Morrison, "Thermodynamic properties of $\mathrm{CH}_{4}$ and $\mathrm{CD}_{4}$. Interpretation of the properties of the solids," The Journal of Chemical Physics, vol. 39, no. 3, pp. 635-653, 1963. 

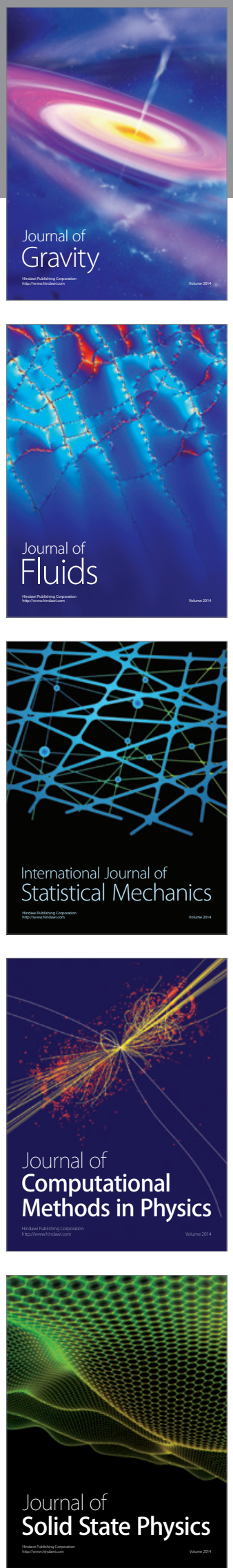

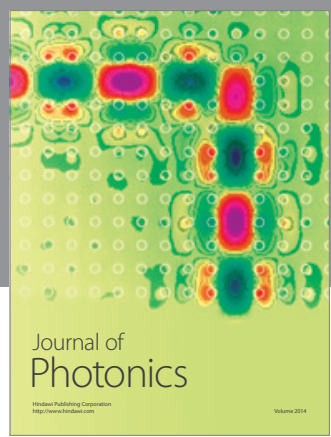

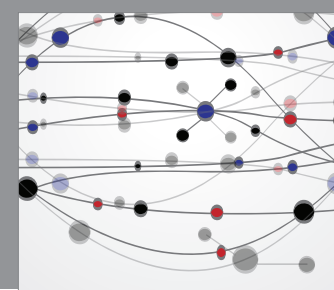

The Scientific World Journal

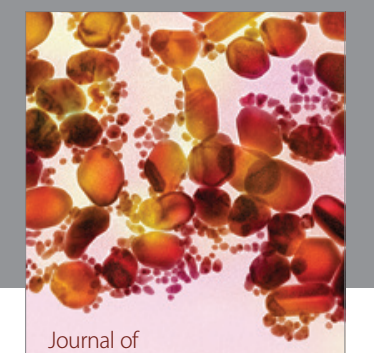

Soft Matter
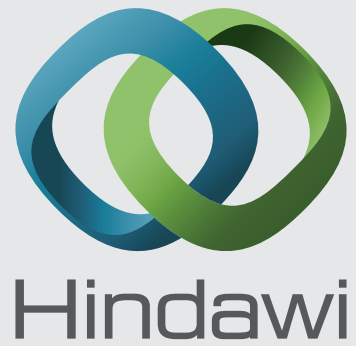

Submit your manuscripts at

http://www.hindawi.com
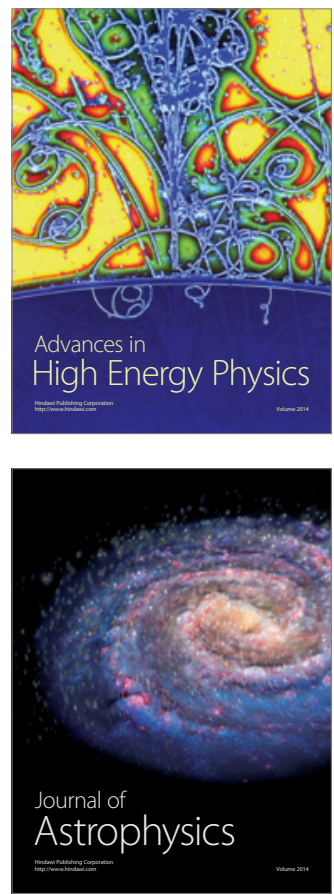
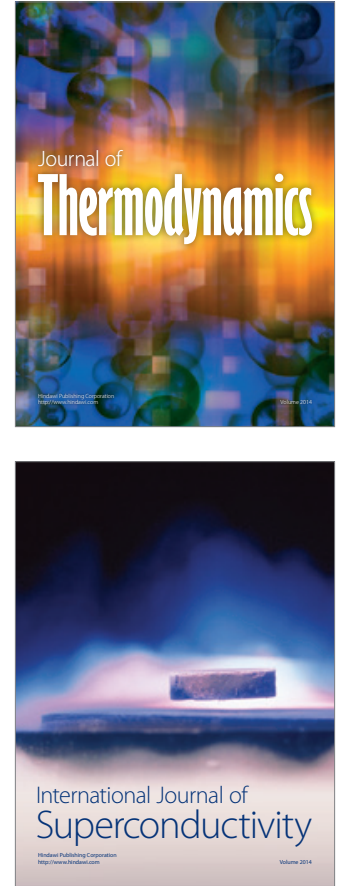
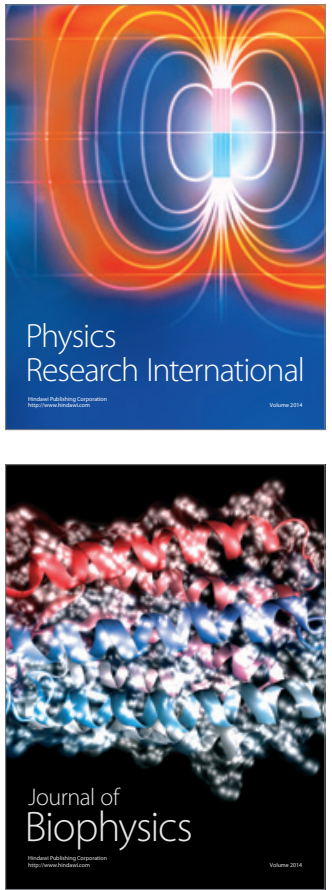
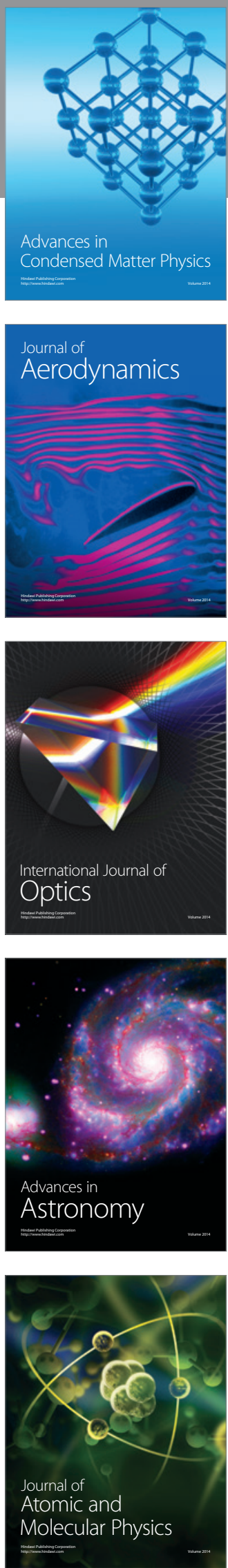